\title{
Examining the provisional guidelines for weight gain in twin pregnancies: a retrospective cohort study
}

Olha Lutsiv ${ }^{1 *}$ (D), Adam Hulman ${ }^{1 \dagger}$, Christy Woolcott ${ }^{2}$, Joseph Beyene ${ }^{3}$, Lucy Giglia ${ }^{4}$, B. Anthony Armson ${ }^{5}$, Linda Dodds ${ }^{2}$, Binod Neupane ${ }^{3}$ and Sarah D. McDonald ${ }^{6}$

\begin{abstract}
Background: Weight gain during pregnancy has an important impact on maternal and neonatal health. Unlike the Institute of Medicine (IOM) recommendations for weight gain in singleton pregnancies, those for twin gestations are termed "provisional", as they are based on limited data. The objectives of this study were to determine the neonatal and maternal outcomes associated with gaining weight below, within and above the IOM provisional guidelines on gestational weight gain in twin pregnancies, and additionally, to explore ranges of gestational weight gain among women who delivered twins at the recommended gestational age and birth weight, and those who did not.
\end{abstract}

Methods: A retrospective cohort study of women who gave birth to twins at $\geq 20$ weeks gestation, with a birth weight $\geq 500 \mathrm{~g}$ was conducted in Nova Scotia, Canada (2003-2014). Our primary outcome of interest was small for gestational age ( $<10$ th percentile). In order to account for gestational age at delivery, weekly rates of 2nd and 3rd trimester weight gain were used to categorize women as gaining below, within, or above guidelines. We performed traditional regression analyses for maternal outcomes, and to account for the correlated nature of the neonatal outcomes in twins, we used generalized estimating equations (GEE).

Results: A total of 1482 twins and 741 mothers were included, of whom 27\%, 43\%, and 30\% gained below, within, and above guidelines, respectively. The incidence of small for gestational age in these three groups was $30 \%, 21 \%$, and $20 \%$, respectively, and relative to gaining within guidelines, the adjusted odds ratios were 1.44 (95\% Cl 1.01-2.06) for gaining below and 0.92 (95\% Cl 0.62-1.36) for gaining above. The gestational weight gain in women who delivered twins at 37-42 weeks with average birth weight $\geq 2500 \mathrm{~g}$ and those who delivered twins outside of the recommend ranges were comparable to each other and the IOM recommendations.

Conclusions: While gestational weight gain below guidelines for twins was associated with some adverse neonatal outcomes, additional research exploring alternate ranges of gestational weight gain in twin pregnancies is warranted, in order to optimize neonatal and maternal outcomes.

Keywords: Guidelines, Pregnancy, Small for gestational age, Twins, Weight gain

\footnotetext{
* Correspondence: olha.lutsiv@mail.mcgill.ca

${ }^{\dagger}$ Equal contributors

${ }^{1}$ Department of Obstetrics and Gynecology, McMaster University, 1280 Main

Street West, Room 3N52B, Hamilton, ON L8S 4K1, Canada

Full list of author information is available at the end of the article
}

(c) The Author(s). 2017 Open Access This article is distributed under the terms of the Creative Commons Attribution 4.0 International License (http://creativecommons.org/licenses/by/4.0/), which permits unrestricted use, distribution, and reproduction in any medium, provided you give appropriate credit to the original author(s) and the source, provide a link to the Creative Commons license, and indicate if changes were made. The Creative Commons Public Domain Dedication waiver (http://creativecommons.org/publicdomain/zero/1.0/) applies to the data made available in this article, unless otherwise stated. 


\section{Background}

Weight gain during pregnancy is increasingly recognized as being a key, modifiable perinatal factor with an important impact on a number of maternal and infant outcomes [1]. Recognizing the importance of gestational weight gain (GWG), in 2009 the US Institute of Medicine (IOM) released a guideline specifying the recommended amounts of weight that women with singleton gestations should gain during their pregnancy, depending on their prepregnancy body mass index (BMI) [2]. Since the research on GWG in multiple gestations was limited, only "provisional" recommendations regarding the optimal GWG in twin pregnancies were released at the time.

Despite accounting for only $3 \%$ of births [3], disproportionately more twins than singletons experience morbidity and mortality, occupying $28 \%$ of neonatal intensive care unit (NICU) days [4-6]. Small for gestational age (SGA; defined as birth weight $<10$ th percentile according to singleton cut-offs) affects more twins (14-20\% of twin births), as does low birth weight (LBW) [5]. Due to the morbidity and mortality associated with small infant size, the current IOM guidelines on GWG are designed to reduce the risk of small infant size as well as preterm birth (PTB). Since the incidence of twin pregnancies is likely to continue to increase due to delayed child bearing and assisted reproductive technologies [7], determining the optimal GWG range for this population is of great importance.

While some raise caution at the fact that excessive weight gain during pregnancy may be detrimental to the mother and her baby [8], others have speculated that the provisional GWG recommendations for twin gestations may not be high enough to prevent LBW [9]. GWG above the IOM guidelines for singleton pregnancies is associated with significant adverse maternal outcomes, including pre-eclampsia and overweight/obesity later in life [10-12], and neonatal outcomes, such as high birth weight, which in turn predisposes them to overweight/ obesity in adolescence $[8,13,14]$. Therefore, the benefits of higher GWG for reducing small infant size in twins need to be offset against the possible adverse effects associated with excessive gain [15].

Although several studies have since attempted to explore the adequacy of the provisional IOM GWG guidelines for twins, no consensus has yet been reached. Due to this, more current, robust research to guide optimal GWG in twin pregnancies has been called for [16].

The objectives of this study were two-fold: 1) to examine the association between the existing provisional IOM GWG guidelines for twin pregnancies and SGA (and other secondary maternal and neonatal outcomes), and 2) to determine the GWG in women who delivered twins at the recommended gestational age and birth weight, using a large cohort separate from that in which the provisional guidelines were estimated, in order to explore similarities and/ or differences in optimal GWG.

\section{Methods \\ Study design and data source}

A retrospective cohort study was conducted of all women who gave birth to twins between January 1, 2003 and December 31, 2014 in Nova Scotia, which is an eastern province of Canada. Data for this study were obtained from the Nova Scotia Atlee Perinatal Database (NSAPD), a validated population-based database that captures information on all births within the province [17-19].

The NSAPD contains maternal and neonatal information on demographics, procedures/ interventions, diagnoses, morbidities and mortality for all pregnancies and births, which is extracted from antenatal and medical charts by trained personnel, using standardized forms. The NSAPD is a valid and reliable database, as confirmed by an ongoing quality assurance program, which carries out periodic abstraction studies.

\section{Inclusion and exclusion criteria}

Women who were between 18 and 45 years of age and gave birth to twins at $\geq 20$ weeks were eligible for study inclusion. Women were excluded if one or both of their infants had a birth weight $<500 \mathrm{~g}$, major congenital anomalies, twin-to-twin transfusion syndrome, or if they were conjoined or monoamniotic. Additionally, mothers of single infants with a co-twin loss, mothers of co-twins of infants lost presumably from pregnancies that started with $>2$ fetuses, and mothers of infants with undetermined, unknown, or missing chorionicity were also excluded. Records with missing data on gestational age at delivery, BMI, or maternal weight at the time of delivery were excluded. Women who were underweight $\left(\mathrm{BMI}<18.5 \mathrm{~kg} / \mathrm{m}^{2}\right)$ were excluded since the current IOM guidelines do not provide GWG recommendations for underweight women with twin pregnancies [2].

\section{Exposure, outcome and other variables of interest}

The primary exposure was maternal GWG, categorized as below, within, or above the IOM provisional guidelines for twin pregnancies, according to the woman's prepregnancy BMI group (normal weight BMI 18.5-24.9 kg/ $\mathrm{m}^{2}$, overweight $25.0-29.9 \mathrm{~kg} / \mathrm{m}^{2}$ or obese $\geq 30.0 \mathrm{~kg} / \mathrm{m}^{2}$ ). Women's total GWG was calculated by subtracting their self-reported pre-pregnancy weight, or if unavailable, their first measured weight, from their last measured weight closest to delivery. The measure of pre-pregnancy weight as reported in the NSAPD is based on the value that is written by the physician on the Nova Scotia Prenatal Record (field: "Pre-Pregnancy Weight"). The NSAPD does not record the specific source of this information, however it is known that the physician can use a pre-pregnancy 
weight as reported by the mother or a weight at the first prenatal visit (which for most women will occur in the first trimester; the NSAPD does not capture the date of this visit).

Gestational age at delivery was based on the last menstrual period or ultrasound. The majority of the women (84.5\%) in the NSAPD have an early-pregnancy ultrasound. In women with both gestational age estimates, $87.2 \%$ of the last menstrual period-based estimates are within the ultrasound-based estimates by $+/-1$ completed week. The total GWG recommendations for twin pregnancies for normal weight, overweight and obese women are: $16.8-24.5 \mathrm{~kg}, 14.1-22.7 \mathrm{~kg}$ and $11.4-19.1 \mathrm{~kg}$, respectively. According to the IOM, the average cumulative weight gains for the first trimester (up to 13 weeks of gestation) in women who deliver twins at the recommended gestational age and birth weight (i.e., gestational ages 37-42 weeks and an average twin birth weight $>2500 \mathrm{~g}$ ) are: $3.6 \mathrm{~kg}, 2.1 \mathrm{~kg}$ and $2.0 \mathrm{~kg}$, respectively, for the three BMI categories. The optimal length of gestation was not defined by the IOM, although they considered a gestational age of 37-42 weeks in their recommendations of total GWG. Since that time practice has shifted to twin delivery during the earlier portion of that range in most instances [20, 21]. For this reason, we assumed that a term twin pregnancy is 38 weeks, and thus the recommended 2nd and 3rd trimester weekly rates of weight gain were calculated for each BMI group according to the formula: (IOM recommended total GWG - IOM average cumulative GWG up to 13 weeks)/ (38 weeks 13 weeks). A similar formula was applied to estimate each woman's actual 2nd and 3rd trimester weekly rate of GWG for her BMI group. The women's actual 2nd and 3rd trimester weekly rates of GWG were compared with the calculated IOM recommended rates of gain for their BMI group, and accordingly, women were categorized as gaining below, within, or above the guidelines.

Our primary outcome was SGA, defined as birth weight $<10$ th percentile for gestational age and sex based on singleton growth curves [22]. Singleton growth curves currently provide the best predictors of adverse outcomes in twins, and are thus recommended by obstetrical associations as the preferred method for evaluating growth abnormalities in twins [23]. According to the National Institute for Health and Care Excellence, no evidence-based growth charts specific to twin and triplet pregnancies are available for use [20].

Secondary neonatal outcomes included: birth weight (continuous outcome), LBW $<2500 \mathrm{~g}$, SGA more strictly defined as birth weight $<5$ th percentile for gestational age and sex, large for gestational age (LGA) defined as birth weight $>90$ th percentile for gestational age and sex, Apgar score $<7$ at 5 min, umbilical cord $\mathrm{pH}<7.10$, respiratory distress, hypoglycaemia, NICU admission, and NICU length of stay.

Secondary maternal outcomes included: gestational age at delivery (continuous outcome), PTB $<37$ weeks (overall and broken down into spontaneous and indicated), labour induction, mode of delivery (unassisted birth, instrumental birth, and Caesarean section), postpartum haemorrhage, and length of postpartum hospital stay.

Important covariates included: maternal age, ethnicity, marital status, smoking status and drug use during pregnancy, pre-existing diabetes mellitus, pre-existing hypertension, other physical health problems, mental health problems, prenatal class attendance, chorionicity, and babies' sex. In addition to a woman's level of education, a neighborhood-level measure of socioeconomic status was also included - the Quintile of Adjusted Income Per Person Equivalent (QAIPPE), where 1 corresponds to the lowest and 5 corresponds to the highest income quintiles [24]. Obstetrical history was also included: parity and past history of gestational diabetes, gestational hypertension, LBW, and neonatal death.

\section{Statistical analyses}

Baseline characteristics were compared across the three GWG groups - women who gained below, within, or above the IOM GWG guidelines. Continuous variables were compared with the analysis of variance (ANOVA) or Kruskal-Wallis test. Categorical data were compared with a $X^{2}$ or Fisher's exact test. To address our primary objective, to determine the effects of gaining below, within, or above the IOM GWG guidelines on each of the individual outcomes, linear regression was used for continuous outcomes and logistic regression was used for binary outcomes, with generalized estimating equations (GEE) used for the neonatal outcomes in order to adjust for the correlation between twins in a set. In order to assess the independent effect of GWG on neonatal and maternal outcomes, multivariable analyses were performed, adjusting for baseline maternal and pregnancy characteristics that may confound the associations of interest. The multivariable analyses adjusted for relevant a priori determined variables, which included maternal age, maternal pre-pregnancy BMI, smoking, socioeconomic status, parity, and chorionicity, as well as any additional baseline characteristics significant with a $p$-value $<0.2$ in the univariate analyses. All effect estimates from the models using GEE for the neonatal outcomes and the regression models for the maternal outcomes were reported as mean differences (MD; for continuous outcomes) or odds ratios (OR; for binary outcomes), with their accompanying 95\% confidence intervals $(\mathrm{CI})$. 
Additionally, in order to explore potential GWG effect measure modification by parity or chorionicity, the multivariable models for all maternal and neonatal outcomes were also reanalyzed with an additional interaction term between parity and GWG, and separately chorionicity and GWG. The Wald test was used to test the significance of the interaction terms.

In order to address our second objective and determine the GWG in women who delivered twins at the recommended gestational age and birth weight, we followed the calculations outlined in the IOM guidelines in our study population. The recommended gestational age was defined as birth at 37-42 weeks of gestation. The recommended birth weight was defined as an average twin birth weight $\geq 2500 \mathrm{~g}$. The interquartile range (IQR: 25th and 75th percentiles of the 2nd and 3rd trimester weekly rates of GWG) was determined. To reflect more recent clinical practice, we repeated the calculations after replacing 42 with $38^{6 / 7}$ weeks as the upper limit of gestational age at birth, and further amending the definition of the recommended birth weight, such that both twins individually weighed $\geq 2500$ g. We also determined ranges of GWG in women who delivered twins outside of the recommended gestational age and birth weight, based on both definitions for comparison.

Prior to study commencement, a sample size calculation was performed. Analyses were performed using SAS (version 9.1; SAS Institute Inc., Cary, NC). We followed the Strengthening the Reporting of Observational Studies in Epidemiology (STROBE) guideline [25]. Institutional review board approval was obtained from the Faculty of Health Sciences/ McMaster University Research Ethics Board (\#12-140C), the Reproductive Care Program of Nova Scotia Data Access Committee and the IWK Health Centre Research Ethics Board (\#1012023) prior to study commencement.

\section{Results}

There were 3294 twin hospital births to 1647 women between January 1, 2003 and December 31, 2014 in Nova Scotia (Fig. 1). After excluding records of women who did not meet our study criteria $(N=445)$, and those who had missing information on gestational age, maternal pre-pregnancy height or weight, or maternal weight at delivery $(N=461), 741$ women and 1482 infants were included.

\section{Baseline characteristics}

The majority of the women did not gain the IOM recommended amount of weight during pregnancy; $27.1 \%$ gained below, $29.7 \%$ gained above, and only $43.2 \%$ gained within the guidelines according to the 2nd and 3rd trimester weekly rates of weight gain. The median maternal age was 31 years (IQR 27, 34), with the majority of women being married or common-law (77.8\%), overweight or obese according to their pre-pregnancy BMI classification (26.5\% and $26.3 \%$, respectively), multiparous (52.5\%), and residing in the top two highest neighborhood-level income quintiles (4th and 5th quintile, $23.0 \%$ and $21.0 \%$, respectively). Approximately onefifth of the infants were monochorionic (19.4\%), and $47.2 \%$ were male (with the sex of one baby being ambiguous). Additional baseline characteristics of all study participants are reported in Table 1, according to their IOM classification of GWG.

Women who were excluded from our analyses due to missing information $(N=461)$ had similar baseline characteristics as those included (Additional file 1: Table S1).

\section{Objective 1: Neonatal and maternal outcomes according to IOM classification of GWG}

Our primary outcome, SGA < 10th percentile, occurred in $30.1 \%$ of neonates exposed to inadequate maternal weight gain, which was significantly higher than in neonates exposed to appropriate $(21.1 \%)$ or excess maternal weight gain (19.8\%, Table 2). After controlling for potential confounders, neonates of women who gained below recommendations had a $44 \%$ higher odds of SGA $<10$ th percentile (95\% CI 1.01 to 2.06) than neonates of women who gained within the guidelines (Table 3). They also had higher odds of LBW (adjusted OR 1.55, 95\% CI 1.07 to 2.23 ), and a longer NICU stay (mean +4.4 days, $95 \%$ CI 0.7 to 8.2 days). The adjusted odds of SGA or any secondary neonatal outcome was not significantly different in women who gained above the guidelines compared to those who gained within. Women who gained weight above the provisional guidelines had higher odds of labour induction (adjusted OR 1.65 [95\% CI 1.08, 2.53], Tables 4 and 5).

Gestational age, PTB <37 weeks, instrumental delivery, or postpartum hemorrhage did not differ by groups of GWG. The low incidence of LGA >90th percentile, Apgar score $<7$ at $5 \mathrm{~min}$, and umbilical cord $\mathrm{pH}<7.10$, prohibited their exploration with multivariable regression. We did not find evidence of interaction between the classification of GWG and either parity or chorionicity (i.e., all $p>0.05$ for interaction terms).

\section{Objective 2: GWG in women who delivered twins within and outside the recommended gestational age and birth weight}

Forty-four percent of the women in our study population delivered twins between 37 and 42 weeks, with an average twin birth weight $\geq 2500 \mathrm{~g}$, while $31 \%$ of the women delivered twins between 37 and $38^{6 / 7}$ weeks, with each twin individually having a birth weight $\geq 2500 \mathrm{~g}$. The rates of 2nd and 3rd trimester weight gain appeared to be fairly 


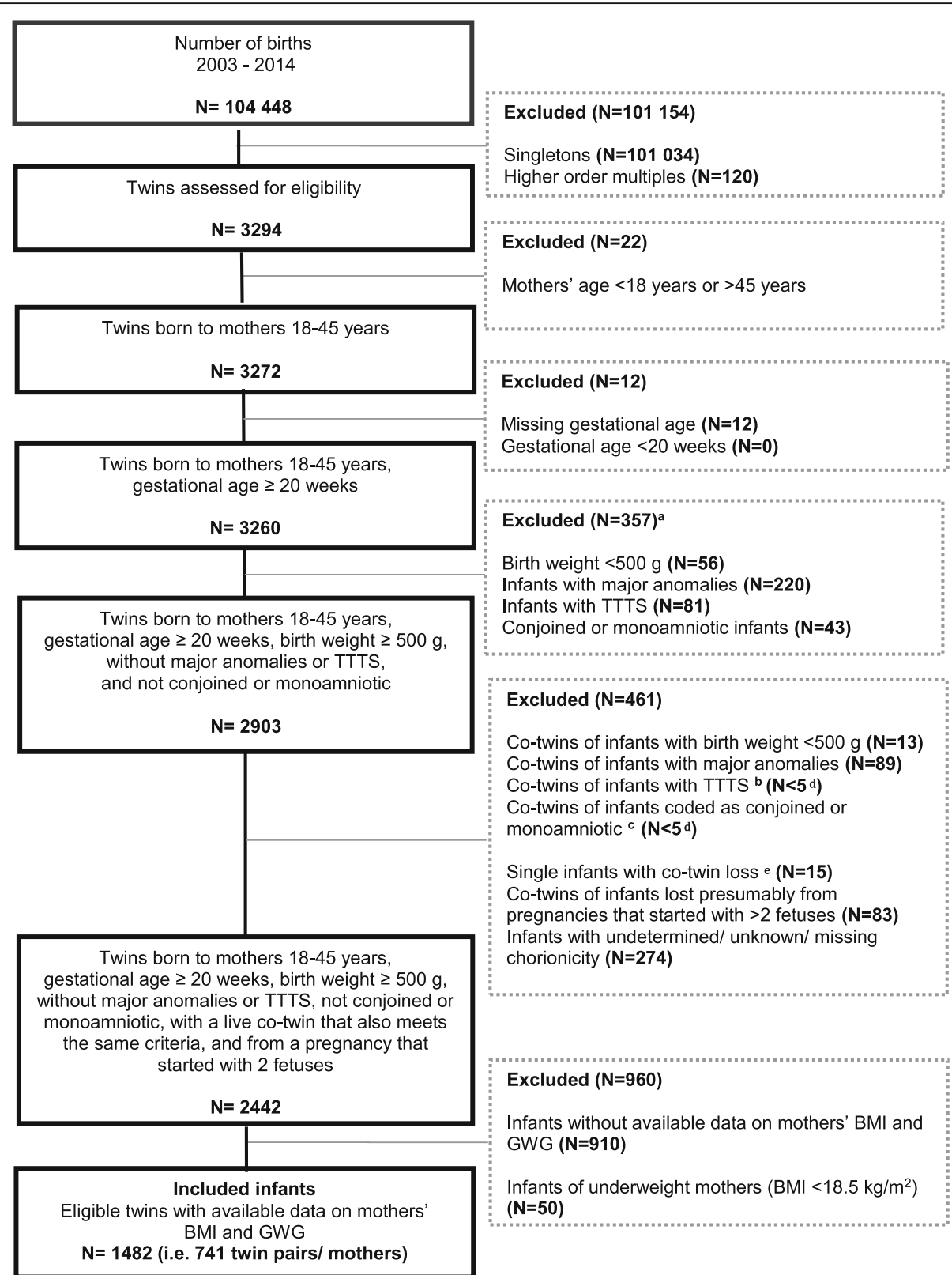

Fig. 1 Flow Chart of Subject Selection. TTTS = Twin Twin Transfusion Syndrome. ${ }^{a}$ some infants excluded for more than one reason. ${ }^{b}$ the co-twin was excluded because they had been coded with TTTS, but the twin that remains in the database was not coded as having TTTS. ${ }^{\complement}$ the co-twin was excluded for having twin type coded as monoamniotic, but the twin that remains in the database was not coded as having this twin type. ${ }^{d}$ exact numbers cannot be reported in order to preserve confidentiality of the research subjects. ${ }^{e}$ of the infants without a co-twin in the dataset, these ones have a code corresponding to continuing pregnancy after spontaneous abortion of one fetus or more, after selective fetal reduction, or after intrauterine fetal demise in other words, ICD10CA codes of O31.11×, O31.12x, or O31.2; there were no instances of O31.0 (papyraceous fetus)

comparable between women who delivered within the recommend gestational age and birth weight ranges and those who did not, regardless of which definition was used, and were also similar to the provisional recommendations from the IOM, regardless of pre-pregnancy BMI (Table 6). Examining the total GWG in women who delivered at the recommended gestational age and birth weight reveals a trend towards higher optimal GWG in normal weight and overweight women compared to the IOM recommendations, but a lower optimal GWG in obese women (Fig. 2).

\section{Discussion}

In this large retrospective cohort study, we determined that relative to gaining within the provisional IOM guidelines for twins, gaining above the guidelines was 
Table 1 Baseline characteristics of women with twin pregnancies according to gestational weight gain

\begin{tabular}{|c|c|c|c|c|c|}
\hline \multirow[t]{2}{*}{ Baseline Characteristic } & \multirow{2}{*}{$\begin{array}{l}\text { All Participants } \\
(N=741) \\
N(\%)^{b}\end{array}$} & \multirow{2}{*}{$\begin{array}{l}\text { Below } 1 O M^{a} \\
\text { GWG } \\
(N=201) \\
N(\%)^{b}\end{array}$} & \multirow{2}{*}{$\begin{array}{l}\text { Within } I^{\mathrm{a}} \\
\text { GWG } \\
(N=320) \\
N(\%)^{b}\end{array}$} & \multirow{2}{*}{$\begin{array}{l}\text { Above } I^{\circ} M^{a} \\
\text { GWG } \\
(N=220) \\
N(\%)^{b}\end{array}$} & \multirow[b]{2}{*}{$P$ value $^{c}$} \\
\hline & & & & & \\
\hline Maternal age, years, median (IQR) & $31(27,34)$ & $29(25,34)$ & $32(28,35)$ & $30(26,34)$ & $<0.001$ \\
\hline Caucasian & $318(82.8)$ & $91(82.0)$ & $134(84.3)$ & $93(81.6)$ & 0.81 \\
\hline Married or common-law & $556(77.8)$ & $145(74.7)$ & $255(82.5)$ & $156(73.6)$ & 0.03 \\
\hline Post-secondary education or higher & $131(50.4)$ & $33(47.1)$ & $66(56.4)$ & $32(43.8)$ & 0.20 \\
\hline Neighborhood-level income quintile & & & & & 0.36 \\
\hline 1st quintile & $139(19.9)$ & $46(23.7)$ & $51(17.1)$ & $42(20.4)$ & \\
\hline 2nd quintile & 109 (15.6) & $33(17.0)$ & $46(15.4)$ & $30(14.6)$ & \\
\hline 3rd quintile & $143(20.5)$ & $38(19.6)$ & $57(19.1)$ & $48(23.3)$ & \\
\hline 4th quintile & $161(23.0)$ & $45(23.2)$ & $70(23.4)$ & $46(22.3)$ & \\
\hline 5th quintile & $147(21.0)$ & $32(16.5)$ & $75(25.1)$ & $40(19.4)$ & \\
\hline Pre-pregnancy BMl, kg/m², median (IQR) & $25.5(22.5,30.3)$ & $26.0(22.6,32.7)$ & $25.0(22.1,29.5)$ & $25.7(23.0,30.1)$ & 0.07 \\
\hline Pre-pregnancy BMI classification & & & & & 0.15 \\
\hline Normal weight (BMI 18.5 to $24.9 \mathrm{~kg} / \mathrm{m}^{2}$ ) & $350(47.2)$ & $93(46.3)$ & $161(50.3)$ & $96(43.6)$ & \\
\hline Overweight (BMI 25.0 to $29.9 \mathrm{~kg} / \mathrm{m}^{2}$ ) & $196(26.5)$ & $45(22.4)$ & $84(26.3)$ & $67(30.5)$ & \\
\hline Obese $\left(\mathrm{BMI} \geq 30.0 \mathrm{~kg} / \mathrm{m}^{2}\right)$ & $195(26.3)$ & $63(31.3)$ & $75(23.4)$ & $57(25.9)$ & \\
\hline Parity $\geq 1$ & $390(52.6)$ & $119(59.2)$ & $172(53.8)$ & $99(45.0)$ & 0.01 \\
\hline Previous gestational diabetes ${ }^{d}$ & $15(2.0)$ & NA & NA & NA & 0.76 \\
\hline Previous gestational hypertension & $29(3.9)$ & $8(4.0)$ & $14(4.4)$ & $7(3.2)$ & 0.78 \\
\hline Previous Caesarean section & $86(11.7)$ & $20(10.1)$ & $40(12.6)$ & $26(12.0)$ & 0.68 \\
\hline Previous LBW ${ }^{d}$ & $20(2.8)$ & NA & NA & NA & 0.31 \\
\hline Previous neonatal death & $7(1.0)$ & NA & NA & NA & 0.31 \\
\hline Smoking during pregnancy & $68(10.3)$ & $30(17.2)$ & $23(7.8)$ & $15(7.9)$ & 0.002 \\
\hline Drug use during pregnancy ${ }^{d}$ & $15(2.0)$ & NA & NA & NA & 0.64 \\
\hline Pre-existing diabetes mellitus ${ }^{d}$ & $9(1.2)$ & NA & NA & NA & 0.002 \\
\hline Pre-existing hypertension ${ }^{d}$ & $15(2.0)$ & NA & NA & NA & 0.32 \\
\hline Other physical health problems & $194(26.2)$ & $57(28.4)$ & $84(26.3)$ & $53(24.1)$ & 0.61 \\
\hline Mental health problems & $69(9.3)$ & $17(8.5)$ & $29(9.1)$ & $23(10.5)$ & 0.76 \\
\hline Attended prenatal classes & $143(41.9)$ & $42(43.3)$ & $50(35.2)$ & $51(50.0)$ & 0.07 \\
\hline Twin type & & & & & 0.52 \\
\hline Monochorionic - diamniotic & $144(19.4)$ & $42(20.9)$ & $52(16.3)$ & $50(22.7)$ & \\
\hline Dichorionic (dissimilar sexes or blood groups) & $294(39.7)$ & $77(38.3)$ & $128(40.0)$ & $89(40.5)$ & \\
\hline Dichorionic (similar sexes and blood groups) & $135(18.2)$ & $34(16.9)$ & $64(20.0)$ & $37(16.8)$ & \\
\hline $\begin{array}{l}\text { Dichorionic (similar sexes but blood groups } \\
\text { undetermined) }\end{array}$ & $168(22.7)$ & $48(23.9)$ & $76(23.7)$ & $44(20.0)$ & \\
\hline Sex of the baby ${ }^{e}$ & & & & & 0.40 \\
\hline Male & $699(47.2)$ & $203(50.5)$ & $294(45.9)$ & $202(46.0)$ & \\
\hline Female & 782 (52.8) & 199 (49.5) & $346(54.1)$ & $237(54.0)$ & \\
\hline
\end{tabular}

Abbreviations: BMI, body mass index; GWG, gestational weight gain; IOM, Institute of Medicine; IQR, inter-quartile range; N, number; NA, not available ${ }^{a}$ GWG categories (below, within, and above IOM GWG) are based on the 2nd and 3rd trimester weekly rates of GWG

${ }^{\mathrm{b}}$ Baseline characteristics are mostly reported as $\mathrm{N}(\%)$, unless otherwise specified (i.e., median (IQR))

${ }^{c} P$ values were calculated with the Kruskal-Wallis test for continuous variables and with the $x^{2}$ test or Fisher's exact test for categorical variables

${ }^{d}$ The proportions of previous gestational diabetes, previous LBW, previous neonatal death, drug use during pregnancy, pre-existing diabetes mellitus, and

pre-existing hypertension could not be reported by GWG category in order to preserve the confidentiality of the research subjects, since some cells had $<5$ events e Sex of the baby is reported at the neonatal level and not the maternal level, thus total $N=1482$; the sex of one baby was ambiguous; the $p$-value is based on the analysis of generalized estimating equations parameter estimates 
Table 2 Outcomes of twins according to mothers' gestational weight gain

\begin{tabular}{|c|c|c|c|c|c|}
\hline \multirow[t]{2}{*}{ Neonatal Outcome } & \multirow{2}{*}{$\begin{array}{l}\text { All Participants } \\
(N=1482) \\
N(\%)^{b}\end{array}$} & \multirow{2}{*}{$\begin{array}{l}\text { Below } \text { IOM }^{\mathrm{a}} \\
\text { GWG } \\
(N=402) \\
N(\%)^{b}\end{array}$} & \multirow{2}{*}{$\begin{array}{l}\text { Within IOM }{ }^{a} \\
\text { GWG } \\
(N=640) \\
N(\%)^{b}\end{array}$} & \multirow{2}{*}{$\begin{array}{l}\text { Above } I^{\circ} \mathrm{M}^{\mathrm{a}} \\
\text { GWG } \\
(N=440) \\
\mathrm{N}(\%)^{\mathrm{b}}\end{array}$} & \multirow[b]{2}{*}{$P$ value ${ }^{c}$} \\
\hline & & & & & \\
\hline SGA $<10$ th percentile & $343(23.2)$ & $121(30.1)$ & $135(21.1)$ & $87(19.8)$ & 0.004 \\
\hline SGA <5th percentile & $193(13.0)$ & $69(17.2)$ & $85(13.3)$ & $39(8.9)$ & 0.005 \\
\hline LBW $<2500 \mathrm{~g}$ & $603(40.7)$ & $204(50.8)$ & $244(38.1)$ & $155(35.2)$ & $<0.001$ \\
\hline LGA >90th percentile & $31(2.1)$ & $5(1.2)$ & $11(1.7)$ & $15(3.4)$ & 0.22 \\
\hline Birth weight, g, median (IQR) & $2603(2260,2885)$ & $2475(2100,2765)$ & $2653(2290,2905)$ & $2637(2356,2910)$ & $<0.001$ \\
\hline Apgar score $<7$ at $5 \mathrm{~min}$ & $39(2.6)$ & $13(3.3)$ & $10(1.6)$ & $16(3.7)$ & 0.16 \\
\hline Umbilical cord pH <7.10 & $33(2.7)$ & $10(2.9)$ & $15(2.8)$ & $8(2.2)$ & 0.84 \\
\hline Respiratory distress & $148(10.0)$ & $46(11.4)$ & $53(8.3)$ & $49(11.1)$ & 0.27 \\
\hline Hypoglycemia & $160(10.8)$ & $43(10.7)$ & $71(11.1)$ & $46(10.5)$ & 0.96 \\
\hline NICU admission & $555(37.5)$ & $170(42.3)$ & $230(35.9)$ & $155(35.2)$ & 0.21 \\
\hline NICU length of stay, days, median (IQR) & $11(2,20)$ & $13(2,23)$ & $10(2,19)$ & $11(1,20)$ & 0.10 \\
\hline
\end{tabular}

Abbreviations: BMI, body mass index; GWG, gestational weight gain; IOM, Institute of Medicine; IQR, inter-quartile range; LBW, low birth weight; LGA, large for gestational age (and sex); N, number; NICU, neonatal intensive care unit; SGA, small for gestational age (and sex)

${ }^{a}$ Gestational weight gain categories (below, within, and above IOM GWG) are based on the 2nd and 3rd trimester weekly rates of GWG

${ }^{b}$ Outcomes are mostly reported as N (\%), unless otherwise specified (i.e., median (IQR))

${ }^{c} P$ values are based on the analysis of generalized estimating equations parameter estimates

associated with higher odds of labour induction, while gaining below was associated with higher odds of SGA, LBW, and longer NICU stay. Additionally, we determined that the rates of 2 nd and 3rd trimester weight gain for women who delivered twins within the recommended gestational age and birth weight ranges, of all pre-pregnancy BMI classes, were fairly comparable to the rates computed based on current recommendations by the IOM, but they were not very different from the rates in women who delivered outside of the recommended gestational age and birth weight ranges.
The original 1990 IOM guidelines recommended 6$20 \mathrm{~kg}$ of GWG for women carrying twins, regardless of their BMI [26]. Given the concerns of small infant size that are associated with low GWG, the IOM revised their guidelines in 2009 to recommend significantly higher GWG (17-25 kg for normal weight women, 14-23 kg for overweight women, and 11-19 kg for obese women) [2]. These guidelines were "provisional", a seemingly reasonable term given that they were based on: a single study; historical in nature (1979 to 1999); with potential for selection bias (four teaching hospitals); and inclusion of

Table 3 Unadjusted and adjusted associations between gestational weight gain and neonatal outcomes

\begin{tabular}{|c|c|c|c|c|}
\hline \multirow{3}{*}{ Neonatal Outcome } & \multicolumn{2}{|l|}{ Below IOM GWG ${ }^{a}$} & \multicolumn{2}{|l|}{ Above IOM GWG ${ }^{a}$} \\
\hline & Unadjusted Analyses & Adjusted Analyses $^{b}$ & Unadjusted Analyses & Adjusted Analyses ${ }^{b}$ \\
\hline & OR $(95 \% \mathrm{Cl})$ & OR $(95 \% \mathrm{Cl})$ & OR (95\% Cl) & OR $(95 \% \mathrm{Cl})$ \\
\hline SGA $<10$ th percentile & $1.61(1.18,2.21)$ & $1.44(1.01,2.06)$ & $0.92(0.66,1.29)$ & $0.92(0.62,1.36)$ \\
\hline SGA $<5$ th percentile & $1.35(0.93,1.98)$ & $1.07(0.69,1.65)$ & $0.64(0.40,1.00)$ & $0.67(0.39,1.14)$ \\
\hline LBW $<2500 \mathrm{~g}$ & $1.67(1.23,2.28)$ & $1.55(1.07,2.23)$ & $0.88(0.65,1.20)$ & $0.81(0.58,1.14)$ \\
\hline Birth weight, $g^{c}$ & $-1523(-235,-71)$ & $-145(-233,-57)$ & $18(-64,100)$ & $39(-48,126)$ \\
\hline Respiratory distress & $1.43(0.87,2.36)$ & $1.14(0.65,2.01)$ & $1.39(0.85,2.27)$ & $1.09(0.63,1.87)$ \\
\hline Hypoglycemia & $0.96(0.60,1.53)$ & $0.73(0.41,1.28)$ & $0.94(0.60,1.46)$ & $1.13(0.69,1.84)$ \\
\hline NICU admission & $1.31(0.94,1.82)$ & $1.29(0.87,1.91)$ & $0.97(0.70,1.35)$ & $0.92(0.63,1.35)$ \\
\hline NICU length of stay, days ${ }^{c}$ & $3.76(0.32,7.21)$ & $4.45(0.69,8.20)$ & $0.92(-2.38,4.22)$ & $1.59(-2.04,5.22)$ \\
\hline
\end{tabular}

Abbreviations: BMI, body mass index; Cl; confidence interval; GWG, gestational weight gain; IOM, Institute of Medicine; IQR, inter-quartile range; LBW, low birth weight; N, number; NICU, neonatal intensive care unit; OR, odds ratio; PTB, preterm birth; SGA, small for gestational age (and sex)

${ }^{a}$ Gestational weight gain categories (below, within, and above IOM GWG) are based on the 2nd and 3rd trimester weekly rates of GWG; GWG within the IOM GWG is the referent group for all analyses

${ }^{b}$ All outcomes were adjusted for the a priori defined confounders, including maternal age, neighborhood-level income, maternal pre-pregnancy BMI, parity, smoking status, and chorionicity. Analyses were also adjusted for the baseline characteristics significant with a $p$-value $<0.2$, including marital status. Despite having a $p$-value $<0.2$, pre-existing diabetes mellitus and attending prenatal classes were not included in the adjusted models, due to low frequency of occurrence in the three categories of GWG (pre-existing diabetes mellitus), or collinearity with other variables in the model (attending prenatal classes)

c The corresponding effect estimates are a mean difference $(95 \% \mathrm{Cl})$, instead of $\mathrm{OR}(95 \% \mathrm{Cl})$ 
Table 4 Outcomes of women with twin pregnancies by gestational weight gain

\begin{tabular}{|c|c|c|c|c|c|}
\hline \multirow[t]{2}{*}{ Maternal Outcome } & \multirow{2}{*}{$\begin{array}{l}\text { All Participants } \\
(\mathrm{N}=741) \\
N(\%)^{b}\end{array}$} & \multirow{2}{*}{$\begin{array}{l}\text { Below } \text { IOM }^{a} \\
\text { GWG } \\
(N=201) \\
N(\%)^{b}\end{array}$} & \multirow{2}{*}{$\begin{array}{l}\text { Within } \mathrm{IOM}^{\mathrm{a}} \\
\text { GWG } \\
(N=320) \\
N(\%)^{\mathrm{b}}\end{array}$} & \multirow{2}{*}{$\begin{array}{l}\text { Above } I^{\circ} M^{a} \\
\text { GWG } \\
(N=220) \\
N(\%)^{b}\end{array}$} & \multirow[b]{2}{*}{$P$ value $^{c}$} \\
\hline & & & & & \\
\hline Gestational age at delivery, weeks, median (IQR) & $37.0(35.7,38.0)$ & $36.9(35.6,38.0)$ & $37.1(35.7,38.0)$ & $37.0(35.7,37.9)$ & 0.49 \\
\hline \multicolumn{6}{|l|}{ PTB $<37$ weeks } \\
\hline Overall & $353(47.6)$ & $104(51.7)$ & $142(44.4)$ & $107(48.6)$ & 0.25 \\
\hline Spontaneous & $155(20.9)$ & $49(24.4)$ & $67(20.9)$ & $39(17.7)$ & 0.25 \\
\hline Indicated & $198(26.7)$ & $55(27.4)$ & $75(23.4)$ & $68(30.9)$ & 0.15 \\
\hline Labour induction & $235(31.7)$ & $67(33.3)$ & $86(26.9)$ & $82(37.3)$ & 0.03 \\
\hline \multicolumn{6}{|l|}{ Mode of delivery } \\
\hline Vaginal birth (unassisted) & $304(41.0)$ & $98(48.8)$ & $125(39.1)$ & $81(36.8)$ & 0.03 \\
\hline Forceps/ vacuum & $50(6.8)$ & $12(6.0)$ & $25(7.8)$ & $13(5.9)$ & 0.60 \\
\hline Caesarean section & $387(52.2)$ & $91(45.3)$ & $170(53.1)$ & $126(57.3)$ & 0.04 \\
\hline Postpartum haemorrhage & $148(20.0)$ & $42(20.9)$ & $63(19.7)$ & $43(19.6)$ & 0.93 \\
\hline Length of stay, days, median (IQR) & $3.4(2.7,4.3)$ & $3.2(2.5,4.1)$ & $3.5(2.8,4.3)$ & $3.6(2.8,4.6)$ & 0.02 \\
\hline
\end{tabular}

Abbreviations: BMI, body mass index; GWG, gestational weight gain; IOM, Institute of Medicine; IQR, inter-quartile range; N, number; PTB, preterm birth

${ }^{a}$ Gestational weight gain categories (below, within, and above IOM GWG) are based on the 2nd and 3rd trimester weekly rates of GWG

${ }^{b}$ Outcomes are mostly reported as $\mathrm{N}(\%)$, unless otherwise specified (i.e., median (IQR))

${ }^{c} P$ values were calculated with the Kruskal-Wallis test for continuous variables and with the $x^{2}$ test or Fisher's exact test for categorical variables

women who delivered between 37 and 42 weeks of gestation, despite the fact that delivery of twins is now recommended between 38 and 39 weeks, to reduce mortality and morbidity [27]; and a focus on twins averaging $\geq 2500 \mathrm{~g}$, thereby precluding the ability to truly examine the association between GWG and individual small infant size [28]. The guidelines did not report on GWG among women with "suboptimal" outcomes (i.e., women who deliver outside of the recommended gestational age and birth weight), even though it is also important to consider

Table 5 Unadjusted and adjusted associations between gestational weight gain and maternal outcomes

\begin{tabular}{|c|c|c|c|c|}
\hline \multirow{3}{*}{ Maternal Outcome } & \multicolumn{2}{|l|}{ Below IOM GWG ${ }^{a}$} & \multicolumn{2}{|l|}{ Above IOM GWG ${ }^{a}$} \\
\hline & Unadjusted Analyses & Adjusted Analyses $^{b}$ & Unadjusted Analyses & Adjusted Analyses ${ }^{b}$ \\
\hline & OR $(95 \% \mathrm{Cl})$ & OR $(95 \% \mathrm{Cl})$ & OR $(95 \% \mathrm{Cl})$ & OR $(95 \% \mathrm{Cl})$ \\
\hline Gestational age at delivery, weeks $^{c}$ & $-0.28(-0.66,0.10)$ & $-0.34(-0.76,0.07)$ & $-0.10(-0.47,0.27)$ & $0.01(-0.39,0.41)$ \\
\hline \multicolumn{5}{|l|}{ PTB $<37$ weeks } \\
\hline Overall & $1.34(0.94,1.91)$ & $1.28(0.86,1.93)$ & $1.19(0.84,1.68)$ & $1.09(0.73,1.62)$ \\
\hline Spontaneous & $1.22(0.80,1.85)$ & $1.16(0.71,1.90)$ & $0.81(0.53,1.26)$ & $0.78(0.47,1.30)$ \\
\hline Indicated & $1.23(0.82,1.84)$ & $1.20(0.76,1.91)$ & $1.46(0.99,2.15)$ & $1.34(0.86,2.09)$ \\
\hline Labour induction & $1.36(0.93,2.00)$ & $1.54(0.99,2.39)$ & $1.62(1.12,2.34)$ & $1.65(1.08,2.53)$ \\
\hline \multicolumn{5}{|l|}{ Mode of delivery } \\
\hline Vaginal birth (unassisted) & $1.48(1.04,2.12)$ & $1.47(0.96,2.25)$ & $0.91(0.64,1.30)$ & $1.03(0.67,1.56)$ \\
\hline Forceps/vacuum & $0.75(0.37,1.53)$ & $0.91(0.40,2.09)$ & $0.74(0.37,1.48)$ & $0.73(0.32,1.67)$ \\
\hline Caesarean section & $0.73(0.51,1.04)$ & $0.71(0.46,1.08)$ & $1.18(0.84,1.67)$ & $1.07(0.71,1.61)$ \\
\hline Postpartum haemorrhage & $1.08(0.70,1.67)$ & $1.10(0.67,1.81)$ & $0.99(0.64,1.53)$ & $1.01(0.62,1.63)$ \\
\hline Length of stay, days ${ }^{c}$ & $-0.30(-0.60,-0.01)$ & $-0.35(-0.68,-0.02)$ & $0.03(-0.25,0.32)$ & $-0.04(-0.36,0.28)$ \\
\hline
\end{tabular}

Abbreviations: BMI, body mass index; Cl; confidence interval; GWG, gestational weight gain; IOM, Institute of Medicine; IQR, inter-quartile range; N, number; OR, odds ratio; PTB, preterm birth

${ }^{a}$ Gestational weight gain categories (below, within, and above IOM GWG) are based on the 2nd and 3rd trimester weekly rates of GWG; GWG within the IOM GWG is the referent group for all analyses

${ }^{b}$ All outcomes were adjusted for the a priori defined confounders, including maternal age, neighborhood-level income, maternal pre-pregnancy BMI, parity, smoking status, and chorionicity. Analyses were also adjusted for the baseline characteristics significant with a $p$-value $<0.2$, including marital status. Despite having a p-value $<0.2$, pre-existing diabetes mellitus and attending prenatal classes were not included in the adjusted models, due to low frequency of occurrence in the three categories of GWG (pre-existing diabetes mellitus), or collinearity with other variables in the model (attending prenatal classes)

c The corresponding effect estimates are a mean difference $(95 \% \mathrm{Cl})$, instead of OR $(95 \% \mathrm{Cl})$ 
Table 6 The 2nd-3rd trimester weekly GWG in women with twin pregnancies with optimal and suboptimal outcomes

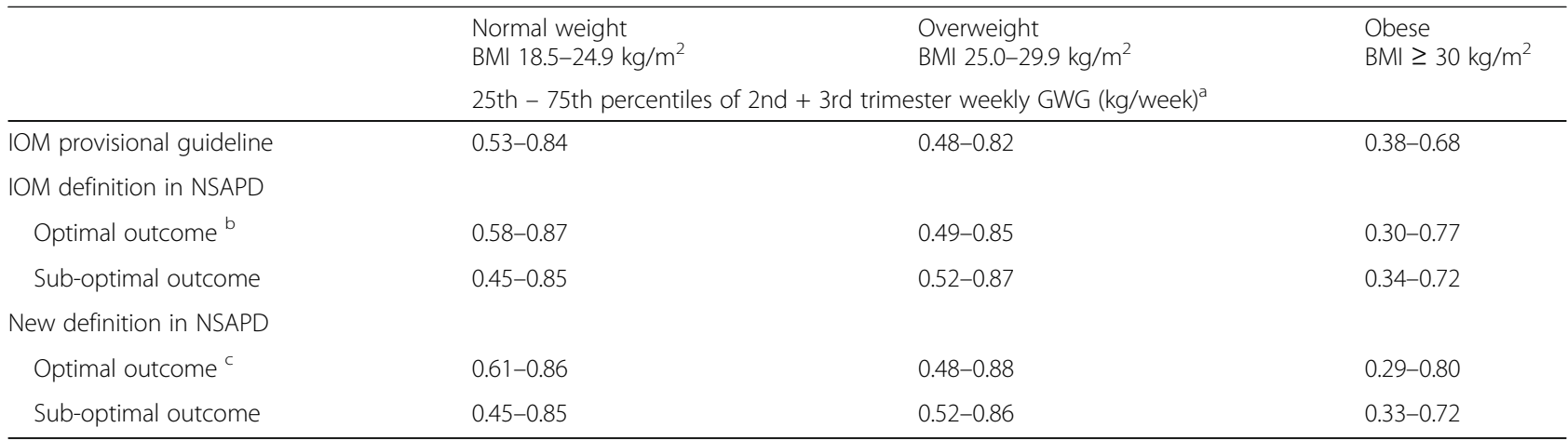

Abbreviations: BMI, body mass index; GWG, gestational weight gain; IOM, Institute of Medicine; NSAPD, Nova Scotia Atlee Perinatal Database

a 2 nd and 3rd trimester weekly GWG were calculated according to the formula: (Total GWG - IOM Average Cumulative GWG up to 13 weeks) / (Gestational Age -

13). The IOM Average Cumulative GWGs up to 13 weeks were $3.6 \mathrm{~kg}, 2.1 \mathrm{~kg}$ and $2.0 \mathrm{~kg}$ in normal weight, overweight and obese women

${ }^{\mathrm{b}}$ Optimal outcome defined as birth at 37-42 weeks and average twin birth weight $\geq 2500 \mathrm{~g}$

c Optimal outcome defined as birth at $37-38^{6 / 7}$ weeks and birth weight of both twins individually $\geq 2500 \mathrm{~g}$

the difference in GWG between women with "optimal" and "suboptimal" outcomes.

Several studies have attempted to examine the 2009 recommendations for twin gestations, with conflicting results. Only a few studies considered SGA $<10$ th percentile specifically, and of those that did, one did not find a significant difference between women [29], while another found a lower incidence in normal weight women who gained within or above guidelines, but no differences in overweight or obese women [9]. A number of studies called into question the provisional recommendations as they found that weight gain in accordance with or in excess of the guidelines was associated with larger birth weight and decreased incidence of prematurity [30-32]. Other studies corroborated these results, as excessive GWG was associated with a larger birth weight, without any significant increases in other adverse pregnancy outcomes [33]. Conversely, another study found both inadequate and excess weight gain were associated with lower birth weight and prematurity [29].

Strengths of this study include the methodology used to classify GWG as below, within, or above guidelines which took into account the length of gestation as recommended by the IOM. Unlike numerous previous studies that restricted their study population to 37 weeks or more, excluding up to $50 \%$ of the population of interest $[30,34]$ and making it impossible to examine "optimal" and "suboptimal" outcomes, or those that assumed a uniform rate of weight gain throughout the pregnancy, our classifications were based on the estimated weekly rates of weight gain for the 2 nd and 3rd trimesters, reflective of the slower trajectory of weight gain during the

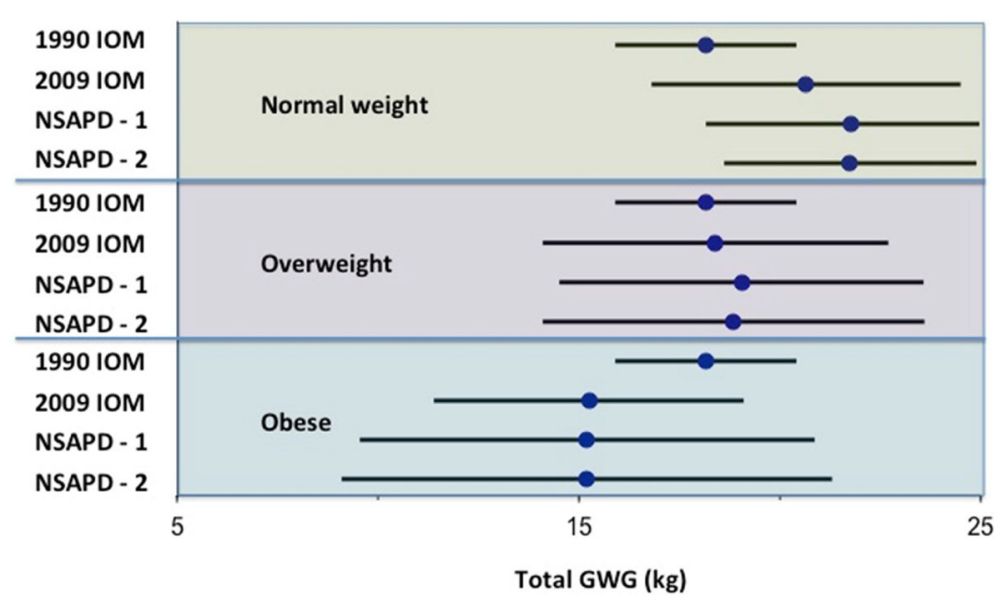

Fig. 2 Total GWG in women with optimal outcomes in NSAPD in comparison to IOM provisional recommendations ${ }^{\mathrm{a}}$. Circle represents the median GWG and the whiskers represent the 25th and 75th percentiles of GWG. Abbreviations: GWG, gestational weight gain; IOM, Institute of Medicine; NSAPD, Nova Scotia Atlee Perinatal Database. a Optimal outcome for NSAPD - 1 defined as birth at 37-42 weeks of gestation and average twin birth weight $\geq 2500 \mathrm{~g}$; Optimal outcome for NSAPD - 2 defined as birth at 37-38 6/7 weeks of gestation, with both twins individually having a birth weight $\geq 2500 \mathrm{~g}$ 
first trimester. This allowed us not only to include a larger sample of women, but much more importantly, did not eliminate women with adverse outcomes, such as PTB. Using a weekly rate of GWG further overcomes some of the bias that can affect results based on total GWG, given its inherent correlation with gestational age. It should be noted, however, that this method may not fully remove the effects of gestational length, as it assumes a certain amount of weight gain in the first trimester; if this assumption is incorrect for some women, it will misclassify them as having inadequate, adequate or excessive weight gain as defined by the IOM provisional guidelines $[35,36]$.

We maintained three BMI groups, rather than grouping together women gaining within and above the guidelines to compare them to women gaining below, which dismisses differences in the incidence of adverse outcomes between the first two groups, and could therefore result in biased effect estimates [30-32]. The large sample size is another notable strength, as it ensured adequate statistical power to control for a number of key confounding variables. Finally, a strength of this study is the use of GEE in order to account for correlation between twins, resulting in robust and valid effect estimates and CIs. Thus we overcame limitations of the majority of previous studies which analyzed all outcomes at the maternal level (instead of the individual neonate level) or which did not account for correlation between twins altogether $[29,31,33]$, which could result in seemingly significant differences between groups even when there are none.

Limitations of this study include some missing data as sometimes occurs with large databases, for some variables of interest, including GWG. However, a comparison of the baseline characteristics of the women who were included in the study and those who were excluded due to missing information did not reveal any significant differences. Furthermore, missing data does not necessarily bias our associations, although our study findings should not be over-generalized to be representative of all pregnant women. Additionally, while our sample size was large enough for us to control for a number of key confounding variables, we were unable to perform analyses stratified by pre-pregnancy BMI, as such analyses would have been underpowered.

Limitations of the method used to develop the provisional guidelines [2] should also be noted. First of all, a comparison of GWG between the women who deliver twins within and outside of the recommended gestational age and birth weight, is an important one, however it is lacking in the IOM guidelines. The smaller the difference between these two groups, the less robust the recommendation. Furthermore, the width of the IOM recommended range of GWG was based on data from a arbitrarily chosen percentiles (25th and 75th) from data from a single study as noted above [28] and not on outcomes. In addition to gestational age and birth weight, other maternal and neonatal outcomes should have also been considered when determining the recommended ranges. Since the currently published studies have mostly aimed to evaluate the provisional guidelines, more attention should be focused on developing new methodologies for defining recommendations, determining whether alternate ranges of GWG correspond with better outcomes, and examining optimal and suboptimal outcomes according to GWG. This is especially critical at this time because if the provisional recommendations are interpreted to be the gold standard, then it will be more difficult to prove that an alternate recommendation may lead to better outcomes, and to change practice.

Future research is required to examine different patterns of GWG throughout pregnancy on maternal and neonatal outcomes, using a longitudinal approach with serial antenatal weight measurements. Determining the optimal GWG for underweight women, and further refining and narrowing the recommended ranges of GWG for normal weight, overweight and obese women is also key. Studying the effects of GWG on SGA defined according to twin growth charts will also be important, if standardized, validated twin growth charts that are based on rigorous data are developed and recommended in clinical practice guidelines.

\section{Conclusions}

In summary, while GWG below the provisional guidelines for twins was associated with SGA and other adverse neonatal outcomes, GWG above the guidelines did not reduce the odds of SGA, and was further associated with adverse maternal outcomes, such as labour induction. As such, GWG recommendations outside of the provisional IOM guidelines may not be advisable, and further research is required to confirm the robustness of the provisional guidelines.

\section{Additional file}

Additional file 1: Table S1. Baseline characteristics of women with twin pregnancies included in analyses and excluded due to missing information. (DOCX $21 \mathrm{~kb})$

\section{Abbreviations}

ANOVA: Analysis of Variance; BMI: Body Mass Index; Cl: Confidence Interval; GEE: Generalized Estimating Equations; GWG: Gestational Weight Gain; IOM: Institute of Medicine; IQR: Inter-Quartile Range; LBW: Low Birth Weight; LGA: Large for Gestational Age; MD: Mean Difference; NICU: Neonatal Intensive Care Unit; NSAPD: Nova Scotia Atlee Perinatal Database; OR: Odds Ratio; PTB: Preterm Birth; QAIPPE: Quintile of Adjusted Income Per Person Equivalent; SGA: Small for Gestational Age; STROBE: Strengthening the Reporting of Observational Studies in Epidemiology 


\section{Acknowledgements}

Not Applicable.

\section{Funding}

This work was supported by an operating grant from the Hamilton Academic Health Sciences Organization Academic Funding Plan (HAHSO AFP) Innovation Fund. Additionally, Dr. Sarah D McDonald is supported by a Tier II Canada Research Chair. The funding agencies had no role in the design of the study, collection, analysis and interpretation of the data, and in writing the manuscript.

\section{Availability of data and materials}

The dataset supporting the conclusions of this article is available in the Nova Scotia Atlee Perinatal Database (NSAPD). The data can not be made publically available as per the agreement with NSAPD.

\section{Authors' contributions}

Each of the bylined authors has made a substantial contribution to the study and has fulfilled the International Committee of Medical Journal Editors criteria for authorship. OL made substantial contributions to the study design, performed the statistical analyses, interpreted the data, and drafted the manuscript. $\mathrm{AH}, \mathrm{JB}$, and $\mathrm{BN}$ made substantial contributions to the study design, analysis and interpretation of data. CW, LG, BAA, and LD made substantial contributions to the study design and interpretation of data. SDM made substantial contributions to the study conception and design, acquisition of data, analysis and interpretation of data. All authors were involved in revising the manuscript critically for important intellectual content, approved the final version to be published, and agreed to be accountable for all aspects of the work in ensuring that questions related to the accuracy or integrity of any part of the work are appropriately investigated and resolved.

\section{Ethics approval and consent to participate}

Ethics approval was obtained from the Faculty of Health Sciences/ McMaster University Research Ethics Board (\#12-140C), the Reproductive Care Program of Nova Scotia Data Access Committee and the IWK Health Centre Research Ethics Board (\#1012023). This study was conducted using anonymized registry data, conforming to the 2014 Tri-Council Policy Statement for ethical conduct of research involving humans in Canada, and thus the need for consent was waived by the research ethics committees.

\section{Consent for publication}

Not Applicable.

\section{Competing interests}

The authors declare that they have no competing interests.

\section{Publisher's Note}

Springer Nature remains neutral with regard to jurisdictional claims in published maps and institutional affiliations.

\section{Author details}

'Department of Obstetrics and Gynecology, McMaster University, 1280 Main Street West, Room 3N52B, Hamilton, ON L8S 4K1, Canada. Departments of Obstetrics and Gynaecology, and Pediatrics, Dalhousie University, Halifax, NS, Canada. ${ }^{3}$ Department of Clinical Epidemiology \& Biostatistics, McMaster University, Hamilton, ON, Canada. ${ }^{4}$ Department of Pediatrics, McMaster University, Hamilton, ON, Canada. ${ }^{5}$ Department of Obstetrics and Gynaecology, Dalhousie University, Halifax, NS, Canada. ${ }^{6}$ Departments of Obstetrics and Gynecology, Radiology, and Clinical Epidemiology \& Biostatistics, McMaster University, Hamilton, ON, Canada.

Received: 8 May 2016 Accepted: 21 September 2017 Published online: 29 September 2017

\section{References}

1. Dzakpasu S, Fahey J, Kirby RS, et al. Contribution of prepregnancy body mass index and gestational weight gain to adverse neonatal outcomes: population attributable fractions for Canada. BMC Pregnancy Childbirth 2015;15:21.
2. Institute of Medicine (IOM). National Research Council. Weight Gain During Pregnancy: Reexamining the Guidelines. In: Rasmussen KM, Yaktine AL, editors. Committee to Reexamine IOM Pregnancy Weight Guidelines. Washington, DC: National Academy Press; 2009.

3. Public Health Agency of Canada. Canadian Perinatal Health Report. 2008. Ottawa.

4. Bassil KL, Shah PS, Barrington KJ, et al. The changing epidemiology of preterm twins and triplets admitted to neonatal intensive care units in Canada, 2003 to 2008. Am J Perinatol. 2012;29(4):237-44.

5. Kogan MD, Alexander GR, Kotelchuck $M$, et al. Trends in twin birth outcomes and prenatal care utilization in the United States, 1981-1997. JAMA. 2000;284(3):335-41.

6. Martin JA, Hamilton BE, Sutton PD, Ventura SJ, Menacker F, Kirmeyer S. Births: final data for 2004. Centers for Disease Control and Prevention, National Center for Health Statistics, National Vital Statistics System. 2006;55(1):1-101.

7. Johnson JA, Tough S. Society of O, Gynaecologists of C. Delayed childbearing. J Obstet Gynaecol Can. 2012;34(1):80-93.

8. Nohr EA, Vaeth M, Baker JL, Sorensen T, Olsen J, Rasmussen KM. Combined associations of prepregnancy body mass index and gestational weight gain with the outcome of pregnancy. Am J Clin Nutr. 2008;87(6):1750-9.

9. Lal AK, Kominiarek MA. Weight gain in twin gestations: are the Institute of Medicine guidelines optimal for neonatal outcomes? J Perinatol. 2015;35(6):405-10

10. Macdonald-Wallis C, Tilling K, Fraser A, Nelson SM, Lawlor DA. Gestational weight gain as a risk factor for hypertensive disorders of pregnancy. Am J Obstet Gynecol. 2013;209(4):327 e1-17.

11. Gunderson EP, Abrams B. Epidemiology of gestational weight gain and body weight changes after pregnancy. Epidemiol Rev. 2000;22(2):261-74.

12. Rooney BL, Schauberger CW, Mathiason MA. Impact of perinatal weight change on long-term obesity and obesity-related illnesses. Obstet Gynecol. 2005:106(6):1349-56

13. Thorsdottir I, Torfadottir JE, Birgisdottir BE, Geirsson RT. Weight gain in women of normal weight before pregnancy: complications in pregnancy or delivery and birth outcome. Obstet Gynecol. 2002;99(5 Pt 1):799-806.

14. Yu ZB, Han SP, Zhu GZ, et al. Birth weight and subsequent risk of obesity: a systematic review and meta-analysis. Obes Rev. 2011;12(7):525-42.

15. McDonald SD, Han Z, Mulla S, et al. High gestational weight gain and the risk of preterm birth and low birth weight: a systematic review and metaanalysis. J Obstet Gynaecol Can. 2011;33(12):1223-33.

16. Leese B, Jomeen J, Denton J. Appropriate maternal weight gain in singleton and twin pregnancies: what is the evidence? Hum Fertil (Camb). 2012;15(4):194-9.

17. A Provincial Program of the Nova Scotia Department of Health and Wellness. The Nova Scotia Atlee Perinatal Database; 2014. (cited 2015 Sep 20). Available from: http://rcp.nshealth.ca/atlee-database.

18. Fair M, Cyr M, Allen AC, Wen SW, Guyon G, MacDonald RC. An assessment of the validity of a computer system for probabilistic record linkage of birth and infant death records in Canada. The Fetal and Infant Health Study Group. Chronic Dis Can. 2000;21(1):8-13.

19. Joseph KS, Fahey J. Canadian Perinatal Surveillance System - Validation of perinatal data in the Discharge Abstract Database of the Canadian Institute for Health Information. Chronic Dis Can. 2009:29(3):96-100.

20. National Institute for Health and Clinical Excellence (NICE). Multiple Pregnancy: The Management of Twin and Triplet Pregnancies in the Antenatal Period. 2011. Manchester, UK

21. Royal College of Obstetricians and Gynaecologists (RCOG). Management of Monochorionic Twin Pregnancy. 2008. London.

22. Kramer MS, Platt RW, Wen SW, et al. A new and improved population-based Canadian reference for birth weight for gestational age. Pediatrics. 2001; 108(2):E35.

23. Morin L, Lim K. Ultrasound in twin pregnancies. J Obstet Gynaecol Can. 2011;33(6):643-56

24. Wilkins R. PCCF + version 5F: User's quide. Ottawa: Statistics Canada; 2010 (cited 2015 July 25). Available from: http://publications.gc.ca/collections/ collection_2016/statcan/CS82-0086-2005-eng.pdf.

25. von Elm E, Altman DG, Egger M, et al. The Strengthening the Reporting of Observational Studies in Epidemiology (STROBE) statement: guidelines for reporting observational studies. Epidemiology. 2007;18(6):800-4.

26. Institute of Medicine (IOM). Subcommittee on Nutritional Status and Weight Gain During Pregnancy. Washington. DC: National Academy Press; 1990 
27. Hartley RS, Emanuel I, Hitti J. Perinatal mortality and neonatal morbidity rates among twin pairs at different gestational ages: optimal delivery timing at 37 to 38 weeks' gestation. Am J Obstet Gynecol. 2001;184(3):451-8.

28. Luke B, Hediger ML, Nugent C, et al. Body mass index-specific weight gains associated with optimal birth weights in twin pregnancies. J Reprod Med. 2003:48(4):217-24.

29. Shamshirsaz AA, Haeri S, Ravangard SF, et al. Perinatal outcomes based on the institute of medicine guidelines for weight gain in twin pregnancies. $J$ Matern Fetal Neonatal Med. 2014;27(6):552-6.

30. Fox NS, Rebarber A, Roman AS, Klauser CK, Peress D, Saltzman DH. Weight gain in twin pregnancies and adverse outcomes: examining the 2009 Institute of Medicine guidelines. Obstet Gynecol. 2010;116(1):100-6.

31. González-Quintero VH, Kathiresan AS, Tudela FJ, Rhea D, Desch C, Istwan N. The association of gestational weight gain per institute of medicine guidelines and prepregnancy body mass index on outcomes of twin pregnancies. Am J Perinatol. 2012;29(6):435-40.

32. Pettit KE, Lacoursiere DY, Schrimmer DB, Alblewi H, Moore TR, Ramos GA. The association of inadequate mid-pregnancy weight gain and preterm birth in twin pregnancies. J Perinatol. 2015;35(2):85-9.

33. Fox NS, Saltzman DH, Kurtz H, Rebarber A. Excessive weight gain in term twin pregnancies: examining the 2009 Institute of Medicine definitions. Obstet Gynecol. 2011:118(5):1000-4.

34. Gavard JA, Artal R. Gestational weight gain and maternal and neonatal outcomes in term twin pregnancies in obese women. Twin Res Hum Genet. 2014;17(2):127-33.

35. Hutcheon JA, Bodnar LM, Joseph KS, Abrams B, Simhan HN, Platt RW. The bias in current measures of gestational weight gain. Paediatr Perinat Epidemiol. 2012;26(2):109-16.

36. Bodnar LM, Hutcheon JA, Parisi SM, Pugh SJ, Abrams B. Comparison of gestational weight gain $z$-scores and traditional weight gain measures in relation to perinatal outcomes. Paediatr Perinat Epidemiol. 2015;29(1):11-21.

\section{Submit your next manuscript to BioMed Central} and we will help you at every step:

- We accept pre-submission inquiries

- Our selector tool helps you to find the most relevant journal

- We provide round the clock customer support

- Convenient online submission

- Thorough peer review

- Inclusion in PubMed and all major indexing services

- Maximum visibility for your research

Submit your manuscript at www.biomedcentral.com/submit 